\title{
Spontaneous lsolated Dissection of lliac Artery Treated with Endovascular Repair: A Case Report
}

\author{
Elisabetta Tanda ${ }^{1,2}$, Genadi G. Genadiev ${ }^{1}$, Sara Zappadu ${ }^{1,2}$, Gabriele De Donno ${ }^{1,2}$, and Stefano \\ Camparini $^{1}$
}

'Vascular Surgery Service, Cardiovascular Department, San Michele Hospital, ARNAS “G. Brotzu”, Cagliari, ${ }^{2}$ Department of Surgery, University of Cagliari, Monserrato, Italy

\begin{abstract}
Spontaneous isolated dissection of the iliac artery (SID-IA) is a rare pathologic condition. The predisposing factors and best treatment strategies are still being debated. We present the case of a 59-year-old male with acute right lower limb ischemia characterized by the sudden occurrence of rest pain, hypoesthesia, and paresis. Angiography showed SID-IA extending down to the femoral bifurcation. The patient had no risk factors for SID-IA; however, he survived an electrocution and had arterial hypertension at admission. Endovascular revascularization was successfully performed, with complete restoration of limb blood flow and remission of symptoms. Follow-up ultrasonography at 1 year confirmed stent patency and absence of clinical symptoms. Endovascular stenting is a good therapeutic option for symptomatic SID-IA without rupture.
\end{abstract}

Key Words: Dissection, Iliac artery, Endovascular technique, Accidental electrocution, Hypertension
Received June 3, 2021

Revised November 23, 2021

Accepted December 9, 2021

Published on December 28, 2021

Corresponding author: Elisabetta Tanda

Vascular Surgery Service, Cardiovascular Department, San Michele Hospital, ARNAS “G. Brotzu”, Piazzale Alessandro Ricchi 1, 09134 Cagliari (CA), Italy

Tel: 39-070539455

Fax: 39-070539458

E-mail: tanda.elisabetta@gmail.com https://orcid.org/0000-0003-4519-506X

Copyright (C) 2021 The Korean Society for Vascular Surgery

This is an Open Access article distributed under the terms of the Creative Commons Attribution Non-Commercial License (http://creativecommons.org/licenses/by-nc/4.0) which permits unrestricted non-commercial use, distribution, and reproduction in any medium, provided the original work is properly cited.

Cite this article; Vasc Specialist Int 2021. https://doi.org/10.5758/vsi.210040

\section{INTRODUCTION}

Spontaneous isolated dissection of the iliac artery (SID-IA) is a rare pathologic condition. Although the predisposing factors are not fully known, traumatic (trauma [1], extreme physical activity [2], pregnancy [3], and iliac catheterization [4]) and non-traumatic causes (Marfan syndrome [5], Ehlers-Danlos syndrome [6], fibromuscular dysplasia [7,8], and cystic medial degeneration [9]) have been described. The best therapeutic strategy for this condition is still being debated. We present a case of right common iliac artery (CIA) dissection extending down to the femoral bifurcation with true lumen (TL) occlusion causing acute limb ischemia that was successfully treated with endovascular stent implantation. The study was approved by the Ethics Committee of Azienda Ospedaliero-Universitaria di Cagliari, Brotzu Hospital (no. PG/2021/8829). Informed consent was obtained from the patient for publication of this case report and accompanying images anonymously.

\section{CASE}

A previously healthy 59-year-old male presented with sudden rest pain, hypoesthesia, and paresis of the right lower limb. At admission an hour after the onset of symptoms, blood pressure was $190 / 100 \mathrm{mmHg}$, pulse rate 100 bpm, respiratory rate 12 breaths/min, and SpO2 96\%. On physical examination, pulses on the right popliteal, anterior tibial, and posterior tibial arteries were absent, and the right foot was cold. The patient reported a medical history of arterial hypertension for 8 years and an episode of $380 \mathrm{~V}$ alternating current electrocution during housework 7 years earlier, with entry point in the right hand and exit point in the right foot, which was not studied with imaging ex- 
aminations. He denied high-level sporting exercises or any other trauma aside from the electrocution. No cases of sudden death or connective tissue disorders were reported in his family, and he denied other risk factors for atherosclerosis, such as smoking, diabetes, and dyslipidemia. He did not receive any medical therapy and, on his own admission, he did not check his blood pressure periodically. Blood tests confirmed the absence of hyperglycemia, hypercholesterolemia, and inflammatory or infectious diseases. A total body computed tomography scan (Fig. 1, 2) detected a dissection with a primary entry tear at the origin of the right $\mathrm{ClA}$ and

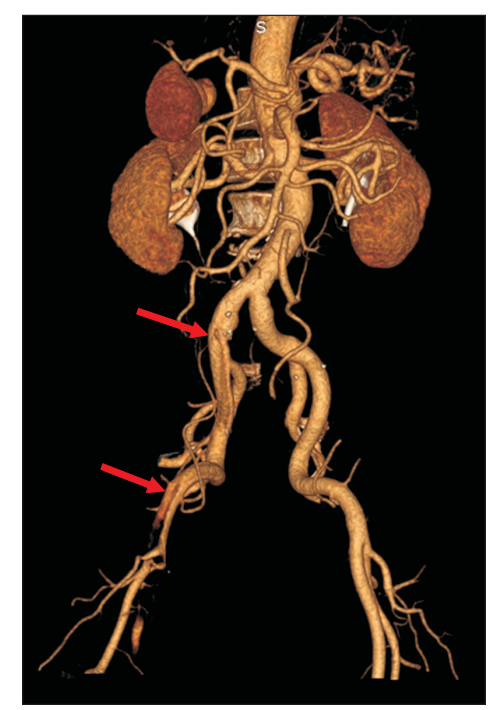

Fig. 1. Computed tomographic reconstruction showed dissection from the origin of the right common iliac artery to the femoral bifurcation. Red arrows indicate the entry sites.

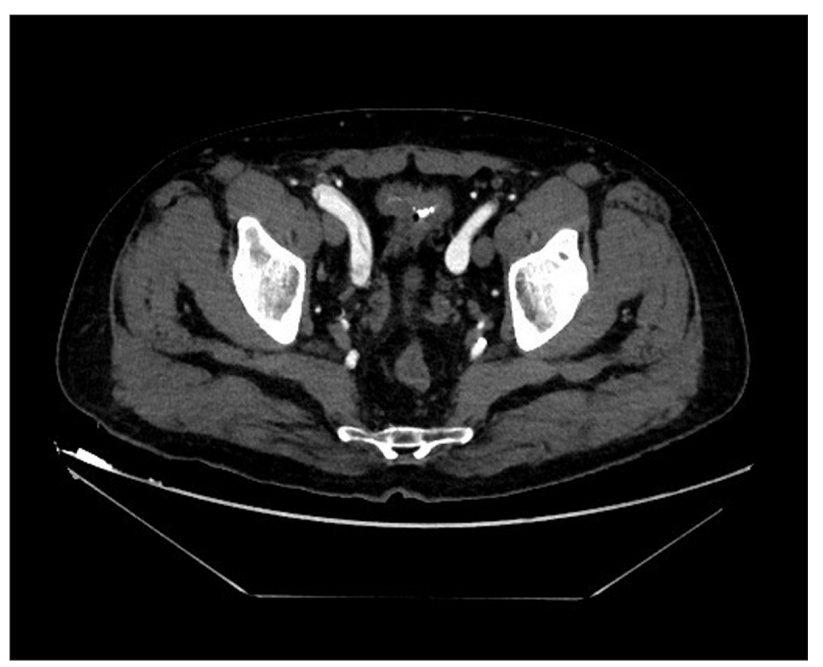

Fig. 2. Cross-sectional images of the computed tomography scan showed the dissection of the right external iliac artery. compression of the TL at the origin of the superficial femoral artery (SFA) with distal collapse, thereby creating an occlusion of the SFA. No atherosclerotic or non-atherosclerotic wall alterations predisposing to dissection, such as the "string of beads appearance of fibromuscular dysplasia", were observed.

The patient underwent angiography (Fig. 3) and endovascular revascularization. The procedure was performed through a contralateral femoral access under local anesthesia. Through a 6-Fr sheath, a hydrophilic Terumo guidewire (Terumo Medical Corporation, Somerset, NJ, USA [10]) was used to cross the TL of the dissected CIA into the SFA. The dissected $\mathrm{ClA}$ and the external iliac artery were treated with 2 self-expanding overlapped stents, E-Luminexx (Becton Dickinson, Franklin Lakes, NJ, USA [11]); the smaller stent (10 $\mathrm{mm} \times 80 \mathrm{~mm}$ ) was first deployed distally while the larger one (12 mm $\times 40 \mathrm{~mm}$ ) was overlapped and deployed using the telescoping technique. The left femoral artery access was closed using a MynxGrip (Cordis, Santa Clara, CA, USA [12]) vascular closure device. Completion angiography showed fully restored blood flow into the right iliofemoral axis (Fig. 4).

No reperfusion compartment syndrome occurred after the procedure. The hospital stay was prolonged due to the patient's high blood pressure, which was successfully managed with olmesartan $20 \mathrm{mg}$ and amlodipine $5 \mathrm{mg}$ once a day. The patient was discharged on the 10th postprocedural day with palpable peripheral pulses. Dual antiplatelet therapy with aspirin and clopidogrel was prescribed for 3 months, followed by lifetime aspirin alone. Follow-up duplex ultrasonography after 1 year confirmed patent stents

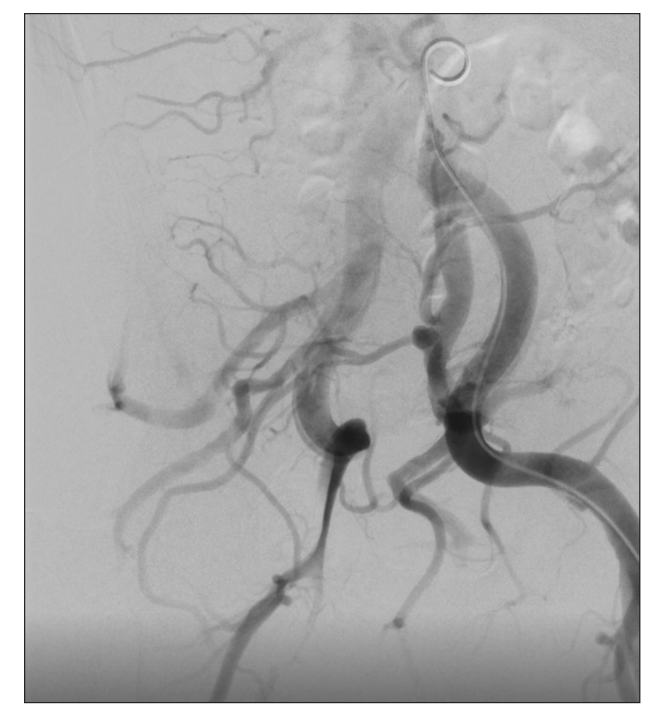

Fig. 3. Diagnostic angiography before the endovascular revascularization confirmed the right iliofemoral dissection. 


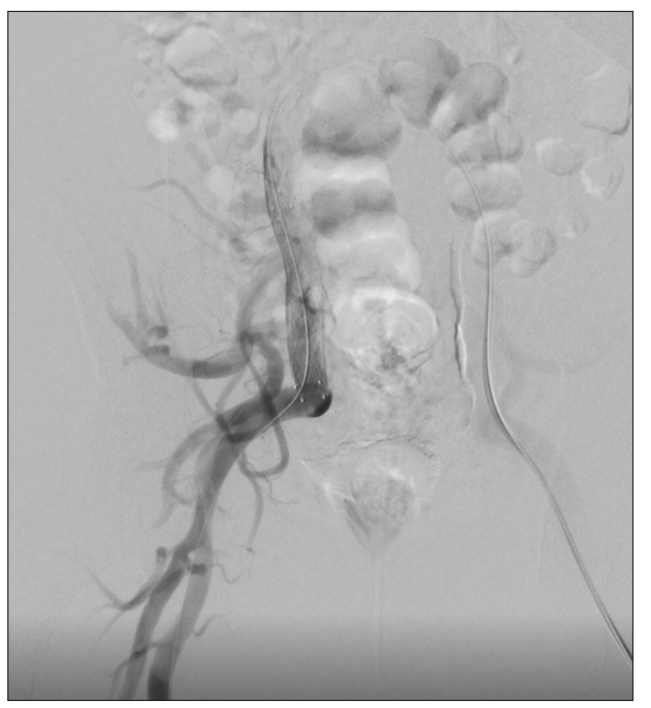

Fig. 4. Completion angiography after stenting showed fully restored blood flow into the right iliofemoral axis. A hydrophilic guidewire was used to cross the true lumen of the dissected common iliac artery into the superficial femoral artery, and 2 stents were successfully deployed.

with good distal blood flow.

\section{DISCUSSION}

SID-IA without involvement of the aorta is extremely rare, and most spontaneous cases are related to connective tissue disorders [5-9] or traumatic causes [1-4]. In the present case, the only predisposing factors identified were hypertension and a previous episode of electrocution. Although arterial hypertension has not been described as a risk factor for SID-IA, it is a known risk factor for aortic dissection [13], predisposing to atherosclerosis and increasing shear stress. Considering that the same mechanisms can potentially act on the iliac arteries as well, we hypothesized that arterial hypertension may have played a role in the occurrence of SID-IA. Moreover, there is evidence that electrocution can lead to vascular injury. Endothelial cell membrane perforation of the aorta and pulmonary artery [14] and a few cases of peripheral arterial rupture (e.g., brachial, radial, ulnar, internal mammary, obturator, and carotid arteries) after electrocution have been reported [15]. In these cases, rupture of the artery immediately followed electrocution. Nonetheless, residual damage caused by electric current may occur in areas of fragility. Therefore, despite the considerable temporal gap with the event in our case, it is not possible to exclude electrocution as a predisposing factor. Our point of view on the risk factors and the suggested treatment concerns a pathology that is still poorly reported in literature because of its rarity. This represents the major limitation of our report, but at the same time, it is what makes it interesting. To our knowledge, this is the first case report of arterial hypertension and electrocution as risk factors for SID-IA and enriches the short list of arterial districts known to have suffered damage from electrocution.

Different strategies have been reported for the management of SID-IA, mainly represented by conservative therapy and endovascular or surgical repair [16]. The clinical presentation usually determines the preferred strategy and timing of intervention. Acute limb ischemia, progressive dissection, and artery rupture are classified as complicated dissection and require urgent intervention $[17,18]$. No guidelines on the treatment of acute ischemia due to iliac dissection are currently available, and the best therapeutic strategies are still being debated, mainly based on evidence derived from aortic dissection. In this context, open surgery (replacement of the dissected aorta with a graft and removal of the intimal tear) is associated with a high incidence of periprocedural complications, such as bleeding, neurological problems, or multiorgan failure, affecting $40 \%$ to $80 \%$ of patients undergoing open repair. Furthermore, open surgery is associated with a non-negligible rate of mortality, with in-hospital mortality rates ranging from $25 \%$ to $50 \%$. On the other hand, the use of endovascular techniques allows less invasive treatment with lower reported periprocedural complications and in-hospital mortality rates (from 2.6\% to 9.8\%) [18]. Endovascular repair has become the firstline therapeutic option in complicated acute type B aortic dissection [16,18], and its successful use with low periprocedural mortality and morbidity has also been reported in SID-IA [16,17].

The primary objectives of the treatment of SID-IA are to limit the extent of dissection, preserve blood flow distally through the TL, and prevent artery rupture. Endovascular repair by covering the primary entry tear can reduce blood pressure within the false lumen (FL), possibly inducing $\mathrm{FL}$ thrombosis and TL re-expansion $[13,18]$, a process called remodeling. This event has the potential to reduce both early and late complications of dissection, including rupture and aneurysmal enlargement [19].

However, some drawbacks of stent implantation must be acknowledged. Stent restenosis, obliteration of side branches [18], and damage to a friable artery with the creation of new entry tears could complicate the procedure, and the implantation could be limited by vessel tortuosity [17]. Moreover, the risk of continued FL perfusion from distal tears must be considered; in these cases, endovascular embolization of the FL can allow successful stent/stent-graft implantation [17].

Another unresolved technical issue involves the length of the artery that should be covered by the endograft $[19,20]$. 
Some studies have shown that a short length of arterial coverage is less likely to result in favorable remodeling. When coverage is too short, the distal end of the stent may damage the friable arterial tissue, creating new entry tears due to movement with the cardiac cycle, while a longer length may physically compress the FL [19]. However, there is no definitive evidence that extended coverage is needed to restore distal perfusion [18] and, traditionally, distal tears remain untreated [19]. We prefer complete re-lining above the inguinal ligament, while we avoid using stents below the ligament if possible because of the change in mechanical characteristics. In summary, our experience confirms that coverage of the primary entry tear may be sufficient to treat the dissection and to induce remodeling observable on follow-up ultrasound as a re-expansion of the TL due to the closure of the entry tear and the consequent pressure drop in the FL. In the iliofemoral axis, we use secondlevel imaging, such as computed tomography, only in case of complications. Considering its low invasiveness, high technical and clinical success rates, and low periprocedural mortality and morbidity [16,17], endovascular treatment could be the preferred therapeutic option in patients with complicated SID-1A without signs of rupture.

\section{FUNDING}

\section{CONFLICTS OF INTEREST}

The authors have nothing to disclose.

\section{ORCID}

\author{
Elisabetta Tanda \\ https://orcid.org/0000-0003-4519-506X \\ Genadi G. Genadiev \\ https://orcid.org/0000-0002-7442-2455 \\ Sara Zappadu \\ https://orcid.org/0000-0001-6684-2639 \\ Gabriele De Donno \\ https://orcid.org/0000-0003-3250-0766 \\ Stefano Camparini \\ https://orcid.org/0000-0002-7202-8729
}

\section{AUTHOR CONTRIBUTIONS}

Concept and design: ET, GGG. Analysis and interpretation: ET, SC. Data collection: none. Writing the article: ET. Critical revision of the article: all authors. Final approval of the article: all authors. Statistical analysis: none. Obtained funding: none. Overall responsibility: all authors.

None.

1) Lyden SP, Srivastava SD, Waldman DL, Green RM. Common iliac artery dissection after blunt trauma: case report of endovascular repair and literature review. J Trauma 2001;50:339-342.

2) Ehsan O, Darwish A, Edmundson C, Mills V, Al-Khaffaf $\mathrm{H}$. Non-traumatic lower limb vascular complications in endurance athletes. Review of literature. Eur J Vasc Endovasc Surg 2004;28:1-8.

3) Nolte JE, Rutherford RB, Nawaz S, Rosenberger A, Speers WC, Krupski WC. Arterial dissections associated with pregnancy. J Vasc Surg 1995;21:515-520.

4) Gul M, Aksu HU, Oz K, Bakir l. Rupture of the NovaFlex balloon during transcatheter aortic valve implantation and subsequent dissection of the right iliac arteries. Eur J Cardiothorac Surg 2013;43:437-438.

5) Barker SG, Burnand KG. Retrograde iliac artery dissection in Marfan's syndrome. A case report. J Cardiovasc Surg (Torino) 1989;30:953-954.

6) Abayazeed A, Hayman E, Moghadamfalahi M, Cain D. Vascular type EhlersDanlos Syndrome with fatal spontaneous rupture of a right common iliac artery dissection: case report and review of literature. J Radiol Case Rep 2014;8:63-69.

7) Honjo O, Yamada Y, Kuroko Y, Kushida Y, Une D, Hioki K. Spontaneous dissection and rupture of common iliac artery in a patient with fibromuscular dysplasia: a case report and review of the literature on iliac artery dissections secondary to fibromuscular dysplasia. J Vasc Surg 2004;40:10321036.

8) Akashi H, Nata S, Kanaya K, Shintani Y, Onitsuka S, Aoyagi S. Spontaneous dissection of the iliac artery in a patient with fibromuscular dysplasia. Ann Vasc Surg 2010;24:952.e13-952. e16.

9) Dueppers P, Jankowiak S, Schelzig $\mathrm{H}$, Wagenhäuser MU, Oberhuber A. Spontaneous rupture of an isolated iliac artery dissection in a young man because of cystic medial degeneration Erdheim-Gsell. Ann Vasc Surg 2015;29:596.e11-596.e13.

10) Niazi K, Farooqui F, Devireddy C, Robertson G, Shaw RE. Comparison of 
hydrophilic guidewires used in endovascular procedures. J Invasive Cardiol 2009;21:397-400.

11) C. R. Bard, Inc. E-luminexx-vascularstents, Instructions for use (IFU), Bard Peripheral Vascular. B05696 Vers.3/1109. Tempe: C. R. Bard, Inc.; 2009.

12) Srivatsa SS, Srivatsa A, Spangler TA. Mynx vascular closure device achieves reliable closure and hemostasis of percutaneous transfemoral venous access in a porcine vascular model. J Invasive Cardiol 2015;27:121-127.

13) Conrad MF, Cambria RP. Aortic dissection: epidemiology, pathophysiology, clinical presentation, and medical and surgical management. In: Sidawy AN, Perler BA, editors. Rutherford's vascular surgery and endovascular therapy. 9th ed. Philadelphia: Elsevier; 2019. p. 1056-1068.
14) Wang $Y$, Liu M, Cheng WB, Li F, Liao Z, Wang Y. Endothelial cell membrane perforation of aorta and pulmonary artery in the electrocution victims. Forensic Sci Int 2008;178:204-206.

15) Toy J, Ball BJ, Tredget EE. Carotid rupture following electrical injury: a report of two cases. J Burn Care Res 2012;33:e160-e165.

16) Liang Z, Guo W, Du C, Xie Y. Effectiveness of the conservative therapy for spontaneous isolated iliac artery dissection: preliminary results. Vascular 2017;25:649-656.

17) Novotny R, Chlupac J, Beran J, Janousek L, Fronek J. Spontaneous isolated common iliac artery dissection treated with self-expandable stent in a 38-year-old patient: a case report. EJVES Short Rep 2018;42:4-6.

18) Riambau V, Böckler D, Brunkwall J,
Cao P, Chiesa R, Coppi G, et al. Editor's choice - management of descending thoracic aorta diseases: clinical practice guidelines of the European Society for Vascular Surgery (ESVS). Eur J Vasc Endovasc Surg 2017;53:4-52.

19) Zhang S, Chen Y, Zhang Y, Shi D, Shen Y, Bao J, et al. Should the distal tears of aortic dissection be treated? The risk of distal tears after proximal repair of aortic dissection. Int J Cardiol 2018;261:162-166.

20) Sayer D, Bratby M, Brooks M, Loftus I, Morgan R, Thompson M. Aortic morphology following endovascular repair of acute and chronic type B aortic dissection: implications for management. Eur J Vasc Endovasc Surg 2008;36:522-529. 